\title{
Hábitos alimentares e comportamento de consumo infantil: influência da renda familiar e do hábito de assistir à televisão ${ }^{1}$
}

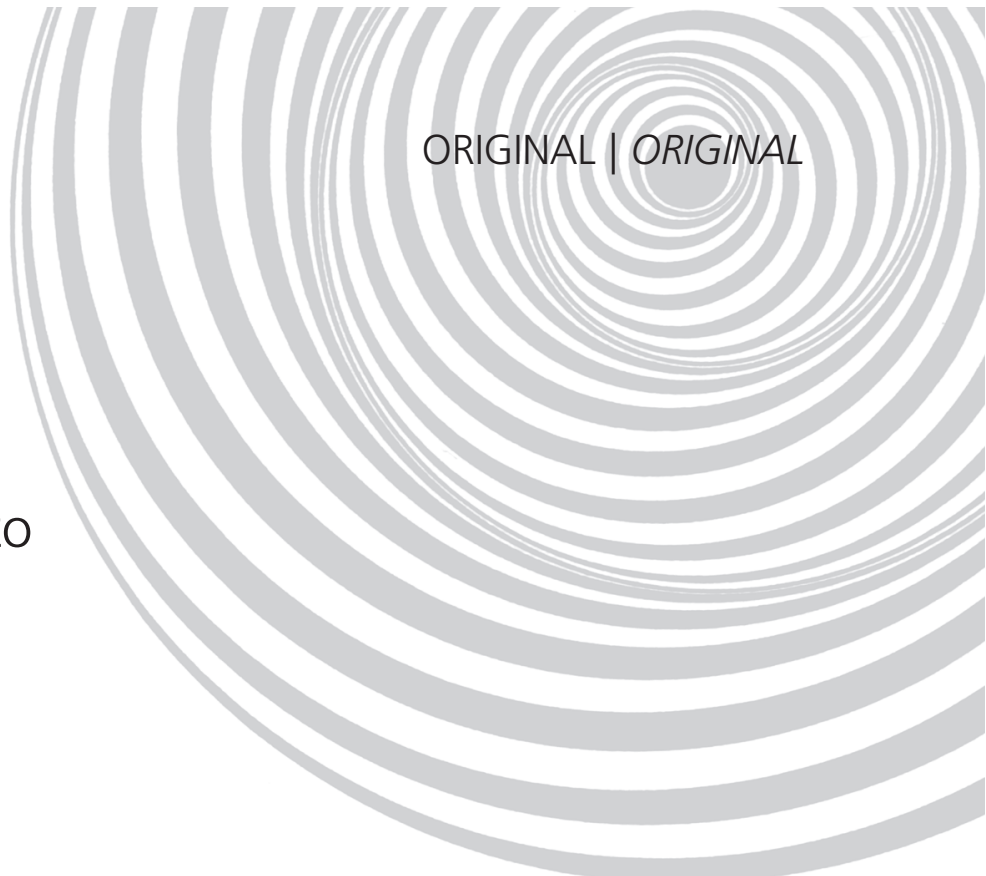

\author{
Children's eating habits and consumer behavior: \\ influence of household income and \\ television viewing habits
}

Vanessa Mello RODRIGUES²

Giovanna Medeiros Rataichesck FIATES²

RE S U M O

\section{Objetivo}

Comparar hábitos alimentares e comportamento de consumo de crianças de diferentes níveis de renda familiar de Florianópolis (SC), Brasil, relacionando-os com o hábito de assistir à televisão.

\section{Métodos}

Estudo qualitativo com análise de conteúdo de manuscritos originados de 23 grupos focais, realizados em uma escola pública e uma escola particular de Florianópolis (SC), compostos por 111 estudantes de 7 a 10 anos. Para verificar a renda familiar dos estudantes, dados sobre a ocupação dos pais foram classificados pela Classificação Brasileira de Ocupações. Os estudantes da escola particular pertenciam a famílias de maior renda em relação aos da escola pública.

\section{Resultados}

Nas duas escolas, a maioria das crianças entrevistadas referiu assistir à televisão sempre que possível, sem sentir controle dos pais sobre esse hábito. Além disso, afirmaram ter dinheiro para gastos independentes e vontade de comprar os produtos anunciados nas propagandas de televisão. Estudantes da escola pública relataram ingerir e adquirir guloseimas mais frequentemente e ter maior liberdade para fazer compras do que os estudantes da escola particular, que revelaram sentir-se controlados pelos pais em relação aos seus hábitos alimentares e compras realizadas.

\footnotetext{
1 Artigo elaborado a partir da dissertação de VM RODRIGUES, intitulada "Hábitos alimentares, comportamento consumidor e hábito de assistir à TV de estudantes de diferentes níveis de renda familiar de Florianópolis". Universidade Federal de Santa Catarina; 2011. Apoio: Programa de Pós-Graduação em Nutrição da Universidade Federal de Santa Catarina, Bolsa do Programa de Apoio ao Plano de Reestruturação e Expansão das Universidades Federais (REUNI) e Coordenação de Aperfeiçoamento de Pessoal de Nível Superior (CAPES).

2 Universidade Federal de Santa Catarina, Centro de Ciências da Saúde, Programa de Pós-Graduação em Nutrição. Campus Universitário Trindade, 88040-900, Florianópolis, SC, Brasil. Correspondência para/Correspondence to: GMR FIATES. E-mail: <gifiates@ccs.ufsc.br>.
} 
354 | VM RODRIGUES \& GMR FIATES

\section{Conclusão}

O fato de os estudantes da escola particular sentirem-se mais controlados por seus pais pode ter reduzido uma provável influência da televisão sobre seus hábitos alimentares e de compras. Evidencia-se a importância da formulação de estratégias para auxiliar os pais a reduzirem os efeitos da televisão sobre os hábitos de seus filhos e de políticas públicas que incentivem o consumo saudável, além da regulamentação do marketing de alimentos pouco nutritivos para o público infantil.

Termos de indexação: Criança. Grupos focais. Hábitos alimentares. Propaganda. Televisão.

\section{A B S T R A C T}

\section{Objective}

This study compared the eating habits and consumer behavior of children from different socioeconomic levels from Florianópolis (SC), Brazil, and investigated their relationship with television viewing habits.

\section{Methods}

The present qualitative study analyzed the transcriptions taken during 23 focus groups done in one public and one private school of Florianópolis (SC). The groups consisted of 111 students aged 7 to 10 years. The students' household income was estimated by parental occupation and classified according to the Brazilian Occupation Classification. The households of private school students had higher income than those of public school students.

\section{Results}

In both schools, most interviewed children reported watching television whenever possible, not perceiving parental control over this habit. Children also mentioned having spending money and the wish to buy products advertised on television ads. The students from the public school reported buying and consuming sweet and savory snacks more often, and having greater freedom to shop than their private school counterparts, who reported feeling greater parental control on their eating and purchase habits.

\section{Conclusion}

The perception of greater parental control may have reduced the influence of television on the spending and eating habits of private school children. The study highlights the importance of formulating strategies that help parents to reduce the impact of television on their children's habits and public policies that encourage healthy food choices. Advertising of unhealthy foods for children should also be regulated.

Indexing terms: Child. Focus groups. Food habits. Advertisement. Television.

\section{N T R O D U ÇÃ O}

Mudanças nos hábitos alimentares de crianças, apesar de diferirem entre os países, seguem uma tendência global na direção de uma alimentação cada vez mais doce e com maior densidade energética, substituindo alimentos ricos em fibras por versões processadas ${ }^{1}$. Além disso, a globalização do fast food e de outros ramos da alimentação moderna está começando a afetar os padrões alimentares infantis de diversos países em transição nutricional².

No Brasil, dados sobre o consumo alimentar de 26 mil crianças de 5 a 10 anos, oriundos do Sistema de Vigilância Alimentar e Nutricional (SISVAN) (2008), indicam consumo inadequado de frutas e, principalmente, de verduras e legumes. Mais de 30\% das crianças não ingeriram esses alimentos durante a semana avaliada. Entretanto, alimentos pouco saudáveis - biscoitos salgados, salgadinhos de pacote, doces e biscoitos recheados - foram consumidos três dias ou mais na semana, por mais de $50 \%$ das crianças $^{3}$.

As crianças são importantes consumidores, pois cada vez mais apresentam recursos próprios para a aquisição de serviços e produtos, principalmente do segmento de alimentos e bebidas. A compra de produtos de maneira independente dos pais acontece em todas as classes sociais ${ }^{4}$. Entretanto, há indícios de que as crianças de menor renda estão tendo maior acesso a produtos menos nutritivos, já que eles estão cada vez mais 
baratos e disponíveis para essa população ${ }^{5}$. Além de populares por sua palatabilidade, alimentos ricos em gordura e açúcar fornecem uma maior oferta de calorias a baixo custo ${ }^{6}$.

Além da renda, a Televisão (TV) também tem sido apontada como um fator que influencia a alimentação, promovendo principalmente hábitos alimentares pouco saudáveis ${ }^{7}$. Essa associação ocorre, entre outras razões, porque na grade de programação televisiva é veiculado um grande número de propagandas de alimentos industrializados e de baixo valor nutricional direcionadas às crianças, o que estimula sua ingestão e aquisição, e diminui o consumo de alimentos saudáveis ${ }^{8}$.

De acordo com o exposto, o objetivo do presente estudo foi comparar hábitos alimentares e comportamento de consumo de crianças de diferentes níveis de renda familiar, e relacioná-los ao hábito de assistir à TV.

\section{M É T O D O S}

Para este estudo, foram utilizadas gravações e transcrições de 23 grupos focais pré-realizadas em uma escola particular (2006) e uma escola pública estadual (2008) de Florianópolis (SC), com crianças de 7 a 10 anos $(n=111)$. As pesquisas conduzidas em ambas as escolas foram aprovadas pelo Comitê de Ética em Pesquisa com Seres Humanos da Universidade Federal de Santa Catarina (projetos n² 293/05 e 171/07), de acordo com o Conselho Nacional de Saúde e com a Declaração de Helsinki. Participaram dos grupos focais somente as crianças cujos responsáveis assinaram o Termo de Consentimento Livre e Es- clarecido, que estiveram presentes nos dias das coletas de dados e que aceitaram participar das entrevistas.

A seleção de uma escola particular e de uma escola pública se deu a fim de diferenciar os níveis de renda familiar das crianças. Para confirmar essa diferença, dados sobre a ocupação dos pais foram coletados das fichas das crianças arquivadas nas escolas e classificados de acordo com a Classificação Brasileira de Ocupações, que organiza as ocupações de forma hierárquica em níveis de complexidade e, consequentemente, remuneração ${ }^{9}$. Dessa forma, evidenciaram-se os diferentes níveis de renda familiar entre as crianças, que na escola particular pertenciam a famílias de maior renda, e, na escola pública, a famílias de menor renda (Tabela 1).

A faixa etária dos participantes do estudo foi escolhida porque nesse período ocorre o desenvolvimento de importantes etapas do comportamento consumidor ${ }^{10}$. Além disso, nessa faixa etária, a criança muitas vezes já realizou o ato da compra independente, sem a interferência dos pais e com seu próprio dinheiro ${ }^{4}$.

Nas duas escolas, foram utilizados guias de discussão semiestruturados para a condução dos grupos focais que se destinavam a conduzir a discussão para temas comuns. No presente trabalho, foram analisados apenas os dados referentes à ingestão de frutas, de legumes, de verduras e de guloseimas, à aquisição de alimentos e ao hábito de assistir à televisão.

Frutas, legumes e verduras foram classificados como alimentos saudáveis, com base na definição do Guia Alimentar para a População Brasileira"1. Foram consideradas "guloseimas" os alimentos que, de acordo com a Lei Estadual

Tabela 1. Total de grupos focais e participantes divididos por idade, sexo e nível de renda familiar. Florianópolis (SC), 2011.

\begin{tabular}{|c|c|c|c|c|c|c|c|c|c|}
\hline & \multicolumn{4}{|c|}{ Idades (anos) } & \multicolumn{2}{|c|}{ Sexo } & \multicolumn{2}{|c|}{ Nível de Renda Familiar } & \multirow{2}{*}{ Total } \\
\hline & 7 & 8 & 9 & 10 & Masculino & Feminino & Maior & Menor & \\
\hline Número de grupos & 4 & 4 & 6 & 9 & 11 & 12 & 12 & 11 & 23 \\
\hline Participantes* & 19 & 21 & 29 & 42 & 52 & 59 & 57 & 54 & 111 \\
\hline
\end{tabular}

*O número de participantes por grupo variou entre 4 e 6 . 
n 12.061 de 2001, estão vetados de comercialização nas cantinas escolares de Santa Catarina (SC). Esses alimentos são: balas, pirulitos e gomas de mascar; refrigerantes e sucos artificiais; salgadinhos industrializados; salgados fritos; e pipocas industrializadas ${ }^{12}$. Além desses, também foram incluídos no grupo alimentos que foram considerados guloseimas pelos próprios entrevistados.

Os manuscritos resultantes dos grupos focais foram submetidos à análise de conteúdo sistemática do tipo dedutiva ${ }^{13}$. A análise dos manuscritos foi feita juntamente com a audição das gravações para detectar as diferentes entonações dadas às falas pelas crianças. Esse processo foi realizado três vezes a fim de permitir a familiarização com o conteúdo dos grupos focais, verificar as repetições de palavras, e identificar os principais temas mencionados. Palavras e frases utilizadas pelos participantes foram analisadas para determinar o grau de similaridade entre as respostas. Os dados foram codificados e, posteriormente, categorizados de acordo com temas comuns. O processo de categorização foi realizado duas vezes pelo mesmo pesquisador, em um intervalo de quinze dias, de modo a assegurar a confiabilidade dos dados ${ }^{14}$. A frequência de comentários foi considerada para mensurar a significância de tópicos específicos, expressa em termos gerais (muitos, poucos, alguns). Alguns comentários foram extraídos das transcrições para ilustrar as categorias originadas.

\section{RES U LTA D OS}

\section{Hábitos alimentares}

As crianças disseram gostar de frutas e consumi-las habitualmente, independentemente do nível de renda, citando as suas favoritas, as mais consumidas e as diversas formas de consumo (sucos, salada de frutas, in natura). Já em relação aos legumes e verduras, grande parte das crianças da escola pública referiu que gostava desses alimentos e que os consumia usualmente, listando os tipos mais consumidos e a frequência de consu- mo. Em contrapartida, em poucos grupos da escola particular apareceram falas nas quais as crianças declararam gostar de legumes e verduras e consumi-los. O consumo de produtos in natura (principalmente legumes e verduras) foi relacionado, por grande parte das crianças da escola pública, à alimentação fornecida nos projetos sociais que frequentavam no contraturno escolar. Nenhuma criança associou o consumo de frutas, legumes e verduras ao ambiente escolar.

Eu gosto de cenoura, beterraba, é... repolho... . Eu como quase todos os dias porque eu vou na Casa da Criança... eu como segunda, terça, quarta, quinta e sexta, e só (Menina, escola pública).

As crianças relataram perceber estímulo por parte dos pais ao consumo de frutas, legumes e verduras. No entanto, entre as crianças da escola particular, foi mais comum a afirmação de que somente comiam esses alimentos por pedido ou exigência dos pais.

Meu pai e minha mãe pedem pra mim comer bastante verdura, mas eu não gosto, só que é tipo assim um acordo: eu como alface e em troca eu não como maçã que eu não gosto (Menina, escola particular).

Quanto ao hábito de consumir guloseimas, a maioria das crianças citou gostar de balas, chocolates, biscoitos, sorvetes, salgadinhos, refrigerantes e frituras. Houve divergência, entretanto, nas respostas relacionadas à frequência de consumo. Enquanto grande parte das crianças da escola particular referiu que só consumia esses alimentos nos finais de semana, a maioria das crianças da escola pública declarou consumi-los diariamente. A maioria das crianças da escola particular relatou que se sentia controlada pelos pais em relação ao consumo de guloseimas, e que o consumo dependia da disponibilidade em casa, o que não ocorria diariamente. Muitos disseram que somente tinham permissão para consumir esses alimentos nos finais de semana ou esporadicamente. 
A minha mãe compra sempre um monte assim de doce e ela deixa dentro do armário. Quando ela dorme eu vou lá no armário e como tudo... ai ela mandou botar fechadura no armário... fez duas cópias de chave (Menino, escola particular).

No entanto, entre as crianças da escola pública, foram feitos poucos comentários sobre restrições ao consumo de alimentos não saudáveis pelos pais, que mencionavam prejuízos à saúde bucal ou gasto desnecessário de dinheiro. Grande parte dessas crianças relatou não perceber controle dos pais sobre seu consumo de guloseimas.

É porque eu peço todos os dias e eles dão... Pirulito, bala, chiclete, salgadinho (Menina, escola pública).

\section{Comportamento de consumo}

A maioria das crianças afirmou ter dinheiro disponível para gastar. As crianças de escola particular disseram receber mesada e presentes em dinheiro dos familiares, enquanto as de escola pública referiram ganhar o troco das compras, trabalhar ou realizar tarefas em casa em troca de dinheiro. As crianças da escola particular afirmaram gastar o seu dinheiro principalmente com brinquedos e jogos eletrônicos. Quando tinham moedas, gastavam com guloseimas. A maioria das crianças da escola pública disse gastar seu dinheiro em guloseimas, além de comprar alimentos para casa ou dar o dinheiro para os pais. Poucos disseram economizar.

Eu gasto quando eu vou no shopping, eu gasto com cinema, brinquedo, roupa assim (Menina, escola particular).

Ontem eu ganhei dois reais, ai hoje eu comprei um monte de coisa. Comprei dois salgadinhos, bala, comprei chocolate (Menina, escola pública).

Em relação às compras realizadas, as crianças de escola pública relataram que comumente faziam compras sozinhas em lugares próximos de casa, como vendinhas ou mercados. Os itens mais relatados como adquiridos para consumo próprio eram guloseimas. Poucas crianças da escola particular relataram ter esse hábito, e disseram fazer compras normalmente com a ajuda dos pais ou irmãos mais velhos, principalmente em relação aos produtos mais caros, ou pedir diretamente aos pais o que queriam.
Eu vou ali na padaria sozinho, e compro sonho, bolacha... . Ela (mãe) me dá, e de vez em quando eu tiro do meu cofrinho (Menino, escola pública).
Então, se eu vejo assim um sapato, que diz que é confortável, bonito e útil, dai eu vou lá, mostro pra minha mãe e a gente vai na loja junto (Menina, escola parti- cular).

\section{Hábito de assistir à televisão}

Falar sobre o hábito de assistir à TV gerou muito interesse entre as crianças, que fizeram comentários entusiasmados, afirmando gostar muito dessa atividade, que é diária. Muitos disseram assistir à TV sempre que possível, nos intervalos de suas atividades, ou durante longos períodos, principalmente à noite.

$$
\begin{aligned}
& \text {...então eu já tenho tipo uma sessão. Fico } \\
& \text { trocando de canal... eu acabo um de- } \\
& \text { senho e já vou lá pra aquele canal que tá } \\
& \text { tendo o outro desenho que eu gosto... } \\
& \text { (Menino, escola particular). } \\
& \text { De manhã eu olho das oito horas que } \\
& \text { começa o desenho, num canal, daí quan- } \\
& \text { do começa no outro canal eu olho daí } \\
& \text { acaba meio-dia. Daí de tarde quando eu } \\
& \text { chego da escola eu olho mais um pouco, } \\
& \text { daí vou tomar banho, daí eu janto, daí } \\
& \text { depois eu olho novela e durmo (Menina, } \\
& \text { escola pública). }
\end{aligned}
$$

Espontaneamente, muitas crianças da escola particular relataram outras atividades de lazer além da TV, como ler ou descer para andar de 
bicicleta, correr e jogar bola nos pátios dos prédios onde residem. Além disso, referiram frequentar diversas atividades fora da escola, como futebol, natação, balé e cursos de línguas. Já entre grande parte das crianças da escola pública, assistir à TV foi apontado como uma das principais opções de lazer.

Poucos comentários foram feitos pelas crianças em relação a restrições dos pais aos horários ou ao conteúdo dos programas de TV. Entre as crianças da escola particular, algumas apontaram que seus pais as proibiam de assistir à TV apenas quando queriam deixá-las de castigo por terem feito algo considerado errado. Já as crianças da escola pública destacaram que o principal conflito relacionado ao hábito de assistir à TV ocorria quando cada pessoa da família queria assistir a um programa diferente e não tinham televisores suficientes para satisfazer a todos. Em relação a comer em frente à TV, de acordo com as crianças, as restrições dos pais eram limitadas à sujeira que poderiam fazer por estarem distraídos enquanto comiam. De modo geral, as crianças demonstraram ter vontade de comprar os produtos anunciados nas propagandas veiculadas na TV, mas citaram principalmente brinquedos.

A propaganda me atrai muito, ela me chama mais atenção do que na própria vitrine da loja (Menina, escola particular).

Eu gosto (de propaganda). Eu fico: ó mãe compra aquilo, ó mãe compra aquilo (Menina, escola pública).

\section{I S C U S S Ã O}

Algumas limitações devem ser consideradas na interpretação dos resultados do presente estudo. A utilização de manuscritos baseados em grupos focais realizados em períodos distintos (2006 e 2008) pode ter influenciado as diferenças observadas entre as respostas das crianças. No entanto, ressalta-se que o processo de transição nutricional vem ocorrendo há cerca de 50 anos $^{15}$ e que nos últimos anos o poder de compra das famílias de classes mais baixas no Brasil cresceu, enquanto o poder de compra da classe média não se alterou substancialmente, o que tornaria os dois grupos mais semelhantes ${ }^{16}$. Uma limitação inerente ao método é o viés da desejabilidade social, que leva alguns indivíduos a darem respostas com a intenção de criar uma imagem específica positiva e supostamente valorizada no seu meio social. O fato de apenas duas escolas terem sido incluídas no estudo pode ser considerado uma limitação adicional, no entanto foram analisados resultados de vinte e três grupos focais envolvendo mais de cem crianças, fato que se reveste de importância quando se considera a escassez de estudos brasileiros que caracterizem o comportamento de consumo de crianças.

No presente estudo, as crianças de escola pública relataram a ingestão mais frequente de frutas e verduras, mas também de guloseimas. Relataram maior liberdade para fazer compras, e para adquirir guloseimas. O hábito de assistir à TV frequentemente foi observado entre a maioria das crianças das duas escolas.

Estudos indicam que, devido ao sabor adocicado, crianças costumam preferir frutas a legumes e verduras ${ }^{17}$. Além disso, a renda tem sido associada ao consumo de frutas, legumes e verduras, pois famílias de baixa renda consomem menores quantidades desses alimentos do que famílias de maior renda ${ }^{18}$. O fato de as crianças da escola pública frequentarem projetos sociais no período contrário à escola também pode ter influenciado no resultado, visto que nesses locais recebiam refeições que incluíam frutas, legumes e verduras. A efetividade de programas não governamentais em comunidades carentes que visem à melhora da alimentação e nutrição entre crianças é reconhecida tanto para a prevenção de doenças como para a promoção de saúde ${ }^{19}$.

Ressalta-se que, em Santa Catarina, a "Lei das Cantinas" 12 determina que se ofereçam duas opções de frutas sazonais diariamente aos alunos, e, de acordo com as normas do Programa Nacional de Alimentação Escolar (PNAE)20, os cardá- 
pios destinados aos alunos da educação básica devem oferecer pelo menos três porções de frutas ou hortaliças por semana. Apesar desses esforços legais para incentivar o consumo de alimentos mais saudáveis dentro do ambiente escolar, a literatura refere que muitas crianças preferem consumir produtos industrializados e lanches rápidos de baixa qualidade nutricional trazidos de casa ${ }^{21}$, o que pode ter ocorrido entre os participantes deste estudo.

Em relação ao consumo de legumes e verduras somente para agradar aos pais, mencionado pelas crianças da escola particular, destaca-se que a imposição às crianças para comer determinados alimentos está associada à diminuição do consumo ou até mesmo à rejeição desses alimentos por elas ${ }^{22,23}$.

O hábito de consumir guloseimas é cada vez mais frequente entre as crianças brasileiras, e tem sido descrito em estudos com diferentes extratos populacionais ${ }^{24}$. A alta palatabilidade desses alimentos já foi apontada como um dos fatores determinantes na escolha das crianças ${ }^{25}$. Entretanto, de modo similar ao observado neste estudo, crianças de famílias com renda mais baixa têm consumido esses alimentos com maior frequência, por seu baixo custo e fácil acesso, principalmente nos pequenos mercados localizados nos bairros mais pobres, nos quais os produtos mais saudáveis costumam ser mais caros e menos disponíveis ${ }^{26}$.

Restringir o acesso a alimentos muito palatáveis pode aumentar nas crianças a preferência por eles, além de despertar o desejo de consumi$-\operatorname{los}^{23}$. No entanto, os filhos de pais mais permissivos tendem a ter hábitos alimentares menos saudáveis ${ }^{27}$. Diante dos diferentes enfoques dados ao modo como os pais devem agir para auxiliar na formação dos hábitos alimentares de seus filhos, observa-se que os melhores resultados são obtidos quando os hábitos e o ambiente familiar servem de base para as práticas alimentares das crianças $^{28}$.

Em relação ao comportamento de consumo, já foi observado que as crianças tinham dinheiro para gastos pessoais, independentemente do nível socioeconômico a que pertencem $^{4}$. Foi possível caracterizar uma situação de maior autonomia das crianças da escola pública como consumidoras, as quais já possuíam mais liberdade para gastar seu dinheiro de maneira independente. Entre as crianças de escola particular, observou-se maior dependência dos pais nas compras.

O gasto com alimentos, principalmente com guloseimas, foi mencionado pela maioria das crianças. Resultados similares já foram destacados por outros estudos latino-americanos, nos quais a maioria das crianças, independentemente da faixa de renda, gastava seu dinheiro com alimentos de baixo valor nutricional29,30.

Entre as crianças da escola pública, esse comportamento foi ainda mais frequente, possivelmente pelo baixo custo de tais produtos e maior disponibilidade nos mercadinhos próximos às suas residências, fato já constatado anteriormente ${ }^{5}$. No Brasil, estudo conduzido com crianças entre 5 e 12 anos de alto e baixo nível socioeconômico também revelou que as meninas mais pobres gastavam mais com guloseimas ${ }^{30}$.

As crianças estão cada vez menos dependentes de seus pais no aprendizado de valores consumidores. A tarefa da instrução tem sido atribuída, entre outros agentes, à TV, atualmente uma das principais fontes de informação sobre novos produtos para crianças ${ }^{7,8}$. Fatores decorrentes da urbanização, como a redução do espaço físico para brincadeiras ao ar livre, o tráfego intenso de veículos, a poluição e a violência nas cidades, além da utilização cada vez mais precoce de meios tecnológicos, favorecem atividades sedentárias como assistir à TV. Entretanto, as pressões comerciais voltadas para essa população e as mensagens cuidadosamente direcionadas a segmentos específicos do público infantil são cada vez mais frequentes $^{8}$. Estudos indicam que as crianças que prestam mais atenção às propagandas de TV compram mais os produtos anunciados ${ }^{19}$. No caso da televisão brasileira, é grande o número de propagandas de alimentos, em sua maioria produtos de baixa qualidade nutricional ${ }^{31}$. 
Apesar de no Brasil ainda não existir recomendação estabelecida, a Organização Mundial da Saúde orienta que crianças não assistam a mais do que duas horas de TV por dia ${ }^{32}$. Desse modo, pode-se dizer que o hábito de assistir à TV, evidenciado pelas falas das crianças, pode ser classificado como excessivo. A regulamentação das propagandas de alimentos de baixo valor nutricional destinadas ao público infantil, que vem sendo discutida constantemente no Brasil, e já existe em grande parte dos países ${ }^{33}$, especialmente na Europa, é importante e deve ser ressaltada.

No presente estudo, as crianças relataram assistir à TV em todos os horários possíveis, não se limitando apenas à programação infantil. Dados de um estudo global revelam que crianças comumente assistem a programas que não foram feitos especificamente para elas, e que, nos horários de maior audiência desse público, o número de propagandas de alimentos pouco saudáveis é ainda maior que durante o resto da programação ${ }^{31}$. A falta de controle dos pais sobre o tempo em que os filhos assistem à TV tem sido relacionada ao baixo nível educacional da mãe ${ }^{34}$ e a hábitos alimentares pouco saudáveis ${ }^{35}$.

\section{O N CLUSÃ O}

Foi possível observar o hábito excessivo de assistir à TV entre os estudantes das duas escolas, sem a percepção do controle dos pais. Entretanto, importantes diferenças nos hábitos alimentares $\mathrm{e}$ no comportamento de consumo foram identificadas entre as crianças dos diferentes níveis de renda. Os estudantes da escola pública relataram a ingestão mais frequente de guloseimas e mais liberdade para fazer compras do que os estudantes da escola particular. É possível que o controle dos pais sobre os hábitos de seus filhos, mais percebido pelos estudantes da escola particular, tenha reduzido uma provável influência da TV sobre os hábitos alimentares e de compra dessas crianças.

Evidencia-se a importância da formulação de políticas públicas que incentivem o consumo de alimentos saudáveis, como, por exemplo, o aumento da tributação sobre produtos industrializados. Ainda, faz-se necessária a consolidação da regulamentação do marketing de alimentos pouco nutritivos direcionados ao público infantil no Brasil, além de estratégias voltadas para auxiliar os pais a reduzirem os efeitos da TV sobre os hábitos de seus filhos.

Por fim, sugere-se a realização de mais estudos que possibilitem refletir e compreender melhor as razões de determinados hábitos e comportamentos infantis e os fatores envolvidos nessas práticas. Propõe-se também a realização de pesquisas que abordem a perspectiva dos pais em relação a tais comportamentos.

\section{COLABORADORES}

VM RODRIGUES participou da análise e interpretação dos dados, redação do artigo e revisão final da versão a ser publicada. GMR FIATES participou da concepção do projeto, revisão crítica relevante do conteúdo intelectual e revisão final da versão a ser publicada.

\section{REFERÊ NCIAS}

1. Popkin B. Global nutrition dynamics: the world is shifting rapidly toward a diet linked with noncommunicable diseases. Am J Clin Nutr. 2006; 84(2):289-98.

2. Adair LS, Popkin BM. Are child eating patterns being transformed globally? Obes Res. 2005; 13(7): 1281-99. doi: 10.1038/oby.2005.153.

3. Brasil. Ministério da Saúde. Coordenação-Geral da Política de Alimentação e Nutrição Análise dos Registros do Consumo Alimentar. Boletim SISVAN n 10. Brasília: MS; 2009 [acesso 2011 jun 1]. Disponível em: <http://nutricao.saude.gov.br/docs/ boletimSisvan/consumo_alimentar_sisvan_ web.pdf>.

4. McNeal JU. Children as consumers of commercial and social products. Washington (DC): PAHO; 2000.

5. Keita AD, Casazza K, Thomas O, Fernandez JR. Neighborhood-level disadvantage is associated with reduced dietary quality in children. J Am Diet Assoc. 2009; 109(9):1612-6. doi: 10.1016/j.jada. 2009.06.373. 
6. Drewnowski A. The role of energy density. Lipids. 2003; 38(2):109-15. doi: 10.1007/s11745-003-10 39-3.

7. Strasburger VC, Jordan AB, Donnerstein E. Health effects of media on children and adolescents. Pediatrics. 2010; 125(4):756-67. doi: 10.1542/peds. 2009-2563.

8. Cairns G, Angus K, Hastings G. The extent, nature and effects of food promotion to children: a review of the evidence to december 2008. Geneva: WHO; 2009.

9. Brasil. Ministério do Trabalho e Emprego. Classificação brasileira de ocupações. Brasília: Ministério do Trabalho e Emprego; 2002 [acesso 2009 out 12]. Disponível em: <http://www.mtecbo.gov.br/ cbosite/pages/informacoesGerais.jsf>.

10. John DR. Consumer socialization of children: a retrospective look at twenty-five years of research. J Cons Res. 1999; 26(3):1-42. doi: 10.1086/20 9543.

11. Brasil. Ministério da Saúde. Secretaria de Atenção à Saúde. Guia alimentar para a população brasileira: promovendo a alimentação saudável. Brasília: MS; 2005.

12. Santa Catarina. Lei $n^{\circ} 12$ 061, de 18 de dezembro de 2001. Dispõe sobre critérios de concessão de serviços de lanches e bebidas nas unidades educacionais, localizadas no Estado de Santa Catarina. Diário Oficial do Estado de Santa Catarina. 2001 20 dez; n 16.810.

13. Bardin L. Análise de conteúdo. Lisboa: Edições 70; 2010.

14. Kidd P, Parshall MB. Getting the focus and the group: enhancing analytical rigor in focus group research. Qual Health Res. 2000; 10(3):293-308. doi: 10.1177/104973200129118453.

15. Batista Filho M, Souza Al, Migliogli TC, Santos MC. Anemia e obesidade: um paradoxo da transição nutricional brasileira. Cad Saúde Pública. 2008; 24(Supl 2):247-57. doi: 10.1590/S0102-311X20 08001400010.

16. Neri MC, coordenador. Evolução dos indicadores sociais baseados na renda: desigualdade de renda na década. Rio de Janeiro: FGV; 2011.

17. Wind M, Bobelijn K, Bourdeaudhij I, Klepp K, Brug J. A qualitative exploration of determinants of fruit and vegetable intake among 10- and 11-year-old schoolchildren in the Low Countries. Ann Nutr Metab. 2005; 49(4):228-35. doi: 10.1159/000 087246.

18. Drewnowski A, Specter E. Poverty and obesity: the role of energy density and energy costs. Am J Clin Nutr. 2004; 79(1):6-16.
19. Alderman $H$, Ndiaye $B$, Linnemayr $S, K a A$, Rokx $C$, Dieng $K$, et al. Effectiveness of a community-based intervention to improve nutrition in young children in Senegal: a difference in difference analysis. Public Health Nutr. 2009; 12(5):667-73. doi: 10.1017/\$13 68980008002619.

20. Brasil. Ministério da Educação. Fundo Nacional de Desenvolvimento da Educação. Lei $n^{\circ} 11.947$, de 16 de Junho de 2009. Dispõe sobre o atendimento da alimentação escolar e do Programa Dinheiro Direto na Escola aos alunos da educação básica; altera as Leis $n^{\circ} 10.880$, de 9 de junho de 2004, $n^{\circ}$ 11.273, de 6 de fevereiro de 2006, n 11.507, de 20 de julho de 2007; revoga dispositivos da Medida Provisória 2.178-36, de 24 de agosto de 2001, e a Lei $n^{\circ} 8.913$, de 12 de julho de 1994; e dá outras providências. Diário Oficial da União. 200917 jun; Seção 1.

21. Matihara $\mathrm{CH}$, Trevisani TS, Garutti S. Valor nutricional da merenda escolar e sua aceitabilidade. Saud Pesq. 2010; 3(1):71-7.

22. Wardle J, Carnell S, Cooke L. Parental control over feeding and children's fruit and vegetable intake: how are they related? J Am Diet Assoc. 2005; 105(1):227-32. doi: 10.1016/j.jada.2004.11.006.

23. Larson N, Story M. A review of environmental influences on food choice. Ann Behav Med. 2009; 18(Suppl 1):56-73. doi: 10.1007/s12160-009-91 20-9.

24. Nunes MM, Figueiroa JN, Alves JGB. Excesso de peso, atividade física e hábitos alimentares entre adolescentes de diferentes classes econômicas em Campina Grande (PB). Rev Assoc Med Bras. 2007; 53(2):130-4. doi: 10.1590/S0104-42302007000 200017.

25. Bower JA, Sandall L. Children as consumers - snacking behaviour in primary school children. Int J Cons Stud. 2002; 26(1):15-26. doi: 10.1046/j.1470-64 31.2002.00220.x.

26. Jetter KM, Cassady DL. The availability and cost of healthier food alternatives. Am J Prev Med. 2006; 30(1):38-44. doi: 10.1016/j.amepre.2005.08.039.

27. Husby I, Heitmann BL, Jensen KO. Meals and snacks from the child's perspective: the contribution of qualitative methods to the development of dietary intervention. Public Health Nutr. 2008; 12(6): 739-47. doi: 10.1017/S1368980008003248.

28. Epstein LH, Dearing KK, Handley EA, Roemmich $J N$, Paluch RA. Relationship of mother and child food purchases as a function of price: a pilot study. Appetite. 2006; 47:115-8. doi: 10.1016/j.appet.20 06.02.005. 
29. Olivares S, Yáñez R, Diaz N. Publicidad de alimentos y conductas alimentarias en escolares de $5^{\circ}$ a $8^{\circ}$ básico. Rev Chil Nutr. 2003; 30(1):36-42. doi: 10.4067/S0717-75182003000100005.

30. Karsaklian E. Comportamento do consumidor. $2^{a}$ ed. São Paulo: Atlas; 2004.

31. Kelly B, Halford JCG, Boyland EJ, Chapman K, Bautista-Castan I, Berg C, et al. Television food advertising to children: a global perspective. Am J Public Health. 2010; 100(9):1730-6. doi: 10.2105/ AJPH.2009.179267.

32. American Academy of Pediatrics. Committee on Public Education. Children, adolescents, and television. Pediatrics. 2001; 107(2):423-6. doi: 10.1542/peds.107.2.423.
33. Hawkes C. Marketing de alimentos para crianças: o cenário global das regulamentações. Brasília: OPAS; 2006.

34. Khawaja M, Barazi R, Linos N. Maternal cultural participation and child health status in a Middle Eastern context: evidence from an urban health study. Child Care Health Dev. 2006; 33(2):117-25. doi: 10.1111/j.1365-2214.2006.00660.x.

35. Dennison BA, Edmunds LS. The role of television in childhood obesity. Prog Pediatr Cardiol. 2008; 25(2):191-7. doi:10.1016/j.ppedcard.2008.05. 010 .

Recebido em: 24/10/2011

Versão final em: 23/4/2012

Aprovado em: 16/5/2012 